\title{
Novel roles for Fe-S clusters in stabilizing or generating radical intermediates
}

\author{
Michael K. Johnson, Christopher R. Staples, Evert C. Duin, Meghan E. Lafferty and \\ Randall E. Duderstadt \\ Department of Chemistry and Center for Metalloenzyme Studies, University of \\ Georgia, Athens, GA 30602, USA.
}

Abstract: The role and properties of the Fe-S clusters in spinach ferredoxinthioredoxin reductase (FTR), $E$. coli biotin synthase and $E$. coli pyruvate formatelyase activating enzyme (PFL-AE) have been investigated by the combination of EPR, resonance Raman, and UV-visible absorption and variable temperature magnetic circular dichroism spectroscopies. FTR is shown to be a novel class of disulfide reductase with an active site involving a [4Fe-4S] cluster and an adjacent cysteine disulfide. The results suggest that the cluster stabilizes the one-electron reduced intermediate of the enzymatic reaction by forming a covalent adduct with one of the cysteines of the active site disulfide, leaving the other cysteine available to form the heterodisulfide adduct with the substrate disulfide. Biotin synthase and PFL-AE are both shown to be homodimeric enzymes with subunit-bridging [ $4 \mathrm{Fe}-4 \mathrm{~S}$ ] clusters that undergo novel $[4 \mathrm{Fe}-4 \mathrm{~S}]^{2+}=2[2 \mathrm{Fe}-2 \mathrm{~S}]^{2+}+2 \mathrm{e}^{-}$cluster conversions. It is proposed that the $[4 \mathrm{Fe}-4 \mathrm{~S}]$ clusters in both enzymes are directly involved with generating a 5 'deoxyadenosyl radical from $S$-adenosyl-L-methionine and that the oxidative cluster conversion provides a means of regulating enzyme activity on exposure to $\mathrm{O}_{2}$ without irreversible cluster degradation. The possibility that the catalytic roles for the [4Fe-4S] clusters in both classes of enzyme investigated in this work involve novel $\mu_{3}-S^{2-}$-based cluster chemistry is discussed.

\section{INTRODUCTION}

In addition to the well-established role in mediating electron transfer, there is increasing evidence that Fe-S clusters serve a wide variety of functions in biology (for recent reviews and specific references, see refs. 1 and 2). They constitute, in whole or in part, the substrate-binding sites of a variety of redox enzymes such as nitrogenase, sulfite reductase, carbon monoxide dehydrogenase, and hydrogenase, and non-redox (de)hydratase enzymes such as aconitase. In order to regulate gene expression or enzyme activity, they appear to act as sensors of iron in the iron responsive protein, oxygen in the fumarate nitrate reduction protein and in glutamine phosphoribosylpyrophosphate amidotransferase, superoxide in the SoxR protein, and nitric oxide in mammalian ferrochelatase. The double-cubane P-clusters and the Rieske-type [2Fe-2S] clusters have also been implicated in coupling proton and electron transfer in nitrogenase and the respiratory/photosynthetic electron transport chains, respectively. In this article, we report on recent spectroscopic studies of representative examples of two new classes of Fe-S-cluster-containing enzymes: a new type of disulfide reductase typified by spinach ferredoxin thioredoxin reductases (FTR) and two new members of the $S$ adenosyl-L-methionine (SAM) dependent class of Fe-S enzymes that function via radical mechanisms, i.e. Escherichia coli biotin synthase and pyruvate formate-lyase activating enzyme. The results suggest new roles for $[4 \mathrm{Fe}-4 \mathrm{~S}]$ clusters in stabilizing or generating radical intermediates and raise the possibility of novel S-based cluster chemistry.

\section{FERREDOXIN THIOREDOXIN REDUCTASE}

FTR is a chloroplast enzyme that plays a key role in the mechanism whereby light regulates the activity of several carbon assimilation enzymes in oxygenic photosynthesis via the cleavage of regulatory disulfides, see Fig. 1 (3). In contrast to the structurally well-characterized flavoprotein disulfide oxidoreductases (4), which 


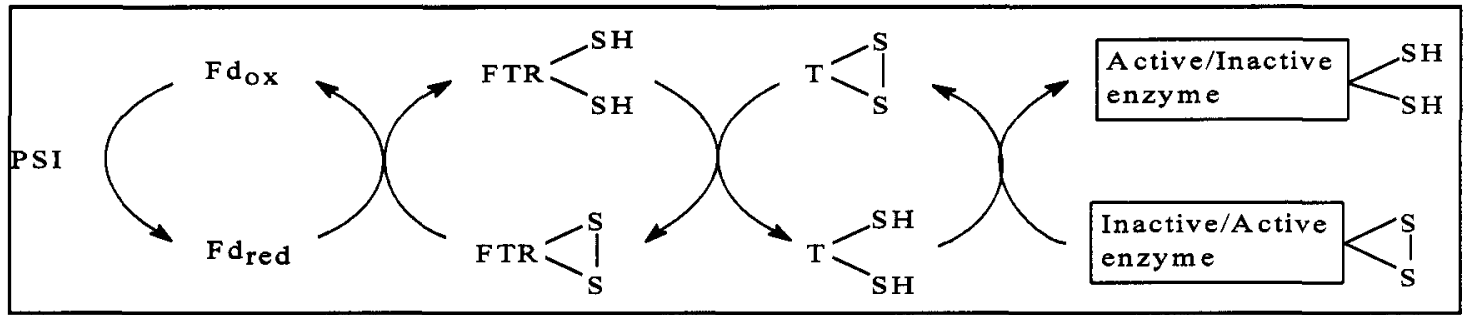

Fig. 1 Role of FTR in the light-mediated regulation of carbon assimilation enzymes in oxygenic photosynthesis

use $\mathrm{NAD}(\mathrm{P}) \mathrm{H}$ as the electron donor and flavin as the electron carrier, the active site disulfide in FTR is cleaved in sequential one-electron steps with chloroplast $2 \mathrm{Fe}$ ferredoxin as the electron donor. The enzyme is a $\alpha \beta$ heterodimer with the $\alpha$ (or variable) subunit having variable size (7-13 $\mathrm{kDa}$ ) and having little or no

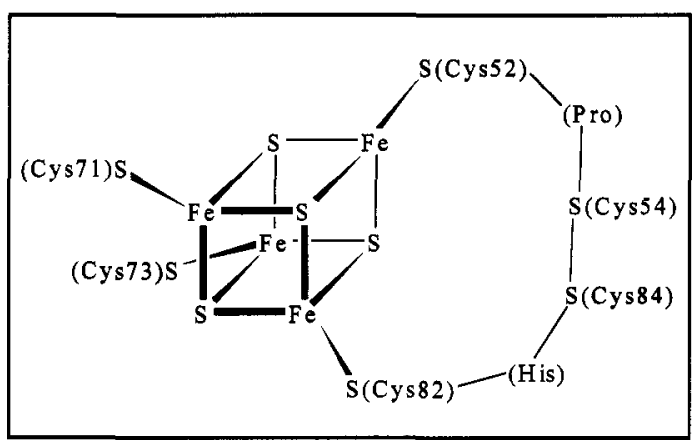

Fig. 2 Schematic picture of the active site in spinach FTR immunological cross reactivity among different species $(5,6)$. In contrast, the $\beta$ (or catalytic) subunit is highly conserved $(13 \mathrm{kDa})(6,7)$ and contains the active site disulfide and a $[4 \mathrm{Fe}-4 \mathrm{~S}]$ cluster $(7,8)$. The role and accessibility of the conserved cysteine residues in the catalytic subunit has been addressed by extensive studies with radiolabeled cysteine alkylating agents and the picture of the active site that emerges from these studies is depicted in Fig. 2 (7). The active-site disulfide is clearly in close proximity to the [4Fe-4S] cluster and, in common with the majority of flavoprotein disulfide reductases (4), only one of the cysteines of the active site disulfide (Cys54) is readily accessible to alkylating reagents such as a $N$ ethylmaleimide (NEM). In the case of flavoprotein disulfide reductases, the other active site cysteine is protected from alkylation by covalent attachment to the flavin isoalloxazine ring (4). As discussed below, the form of the enzyme in which Cys54 is alkylated (termed NEM-FTR) has provided important insights into the mechanism, since it provides a stable analog of the one-electron reduced intermediate in the enzymatic

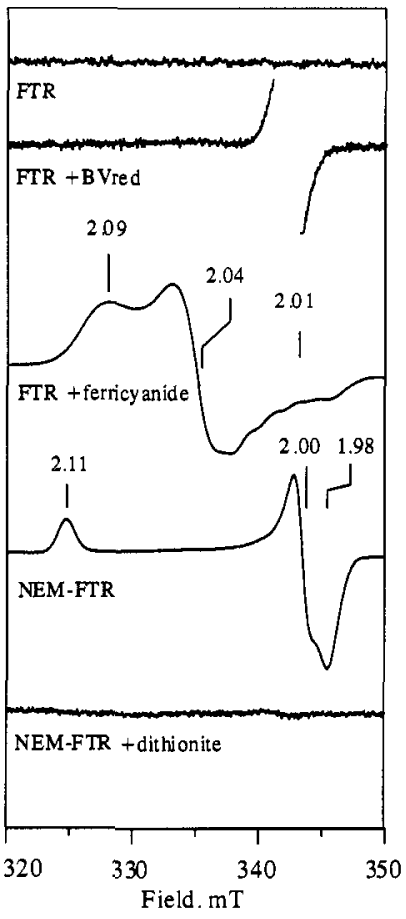

Fig. 3 X-band EPR spectra for FTR $(10 \mathrm{~K}, 10 \mathrm{~mW})$ and NEM-FTR $(35$ $\mathrm{K}, 1 \mathrm{~mW}$ ). mechanism (9).

The nature and properties of the Fe-S cluster in spinach FTR and NEM-FTR have been investigated by the combination of EPR, UV-visible absorption and variable temperature magnetic circular dichroism (VTMCD), and resonance Raman (RR) spectroscopies (8). Figure 3 illustrates the dramatic differences in the EPR properties. As prepared, FTR does not exhibit an EPR signal and (RR) studies clearly demonstrate that the cluster is present as a $S=0[4 \mathrm{Fe}-4 \mathrm{~S}]^{2+}$ center (8). The cluster is not reducible to a paramagnetic $[4 \mathrm{Fe}-4 \mathrm{~S}]^{+}$form using dithionite, reduced benzyl viologen or deazaflavin-mediated photoreduction, indicating a midpoint potential $<-600$ $\mathrm{mV} v \mathrm{sHE}$. Since reduced benzyl viologen, but not dithionite alone, is able to reduce the active site disulfide (10), we conclude that the reducibility of the cluster is not dependent on redox state of the active site disulfide. However, the cluster can be oxidized with ferricyanide to the $[4 \mathrm{Fe}-4 \mathrm{~S}]^{3+}$ state, which exhibits a fast relaxing $S=1 / 2$ EPR signal $(g=2.09,2.04,2.01$ observable at temperatures $<30 \mathrm{~K}$ ), and the midpoint potential for this couple is estimated at $+420 \mathrm{mV} v s$ NHE. This redox process is unlikely to be physiologically relevant, since the midpoint potential is $>600 \mathrm{mV}$ higher than that of the electron donor, spinach ferredoxin $\left(E_{\mathrm{m}(\mathrm{n}=1)}=-420 \mathrm{mV}\right)$ or the active site disulfide $\left(E_{\mathrm{m}(\mathrm{n}=2)}=-230 \mathrm{mV}\right)(11)$. In contrast, NEM-FTR as prepared exhibits a slow relaxing $S=1 / 2$ EPR signal $(g=2.11,2.00,1.98$ and observable without broadening up to at least $150 \mathrm{~K}$ ) that accounts for 1.0 spins/molecule. This resonance is lost on addition of dithionite and EPRmonitored dye-mediated redox titrations indicate one-electron reduction with a midpoint potential of $-210 \mathrm{mV} v \mathrm{~s} \mathrm{NHE}$. 
The significance of the EPR signal associated with NEM-FTR only became apparent as a result of freezequench EPR studies of FTR under turnover conditions (9). Samples of native FTR, containing benzyl viologen and excess thioredoxin $f$, frozen within 5 seconds of initiating the reaction with excess dithionite, exhibit an EPR signal identical to that of NEM-FTR. The EPR signal accounts for $<0.1$ spins/molecule and is lost once all the substrate has been reduced. Subsequent experiments showed that the same EPR signal with a similar spin quantitation is also observed in samples reduced with stoichiometric amounts of reduced benzyl viologen, but disappears on addition of $>2$ equivalents (9). These results indicate that NEM-FTR is a stable analog of the one-electron reduced enzymatic intermediate and offer the prospect of understanding the catalytic mechanism via detailed spectroscopic characterization of NEM-FTR.

In addition to EPR, NEM-FTR has been investigated in both as prepared and dithionite-reduced forms by UVvisible absorption, VTMCD and RR (8). All three techniques point to the same conclusion, namely that NEMFTR contains a $S=1 / 2[4 \mathrm{Fe}-4 \mathrm{~S}]^{3+}$ cluster that is reduced by dithionite to a $S=0[4 \mathrm{Fe}-4 \mathrm{~S}]^{2+}$ cluster. The major differences compared to the $S=1 / 2[4 \mathrm{Fe}-4 \mathrm{~S}]^{3+}$ centers in high potential iron-sulfur proteins (HiPIPs) are the low midpoint potential for one-electron reduction (at least $500 \mathrm{mV}$ lower than in HiPIPs) and the anomalously slow relaxation properties of the EPR resonance. While the EPR relaxation behavior might be construed as being indicative of radical species, the observation of intense temperature dependent MCD bands and the changes in Fe-S vibrational frequencies associated with oxidation and reduction provide clear evidence that redox chemistry is primarily cluster-based and that the unpaired electron spin of NEM-FTR is located on the $\mathrm{Fe}-\mathrm{S}$ cluster rather than a nearby radical species. This conclusion is further substantiated by recent ${ }^{\mathrm{I}} \mathrm{H}-,{ }^{13} \mathrm{C}-$, and ${ }^{57} \mathrm{Fe}-\mathrm{ENDOR}$ studies (9).

The results of the spectroscopic studies of FTR and NEM-FTR in terms of the formal core oxidation states of the $[4 \mathrm{Fe}-4 \mathrm{~S}]$ cluster are summarized in Fig. 4. This begs the question of how one-electron reduction of FTR leads to an intermediate species in which the cluster has been formally oxidized by one electron. Such anomalous redox behavior can only be rationalized by invoking two-electron reduction of the active-site

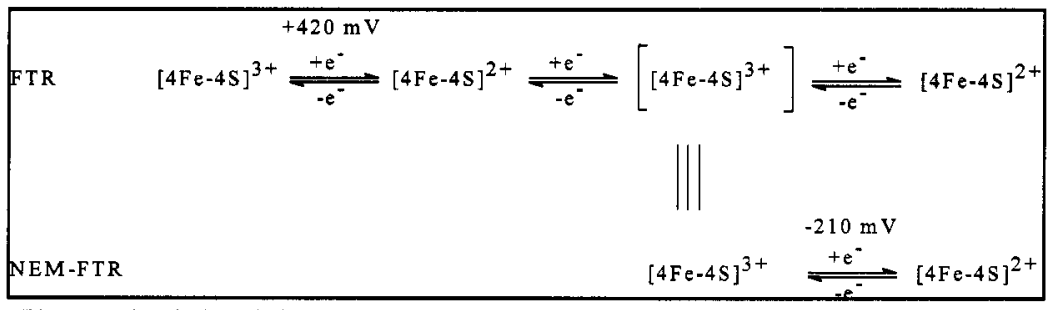

Fig. 4 Redox-induced changes in the core oxidation states of the [4Fe-4S] cluster in FTR and NEM-FTR

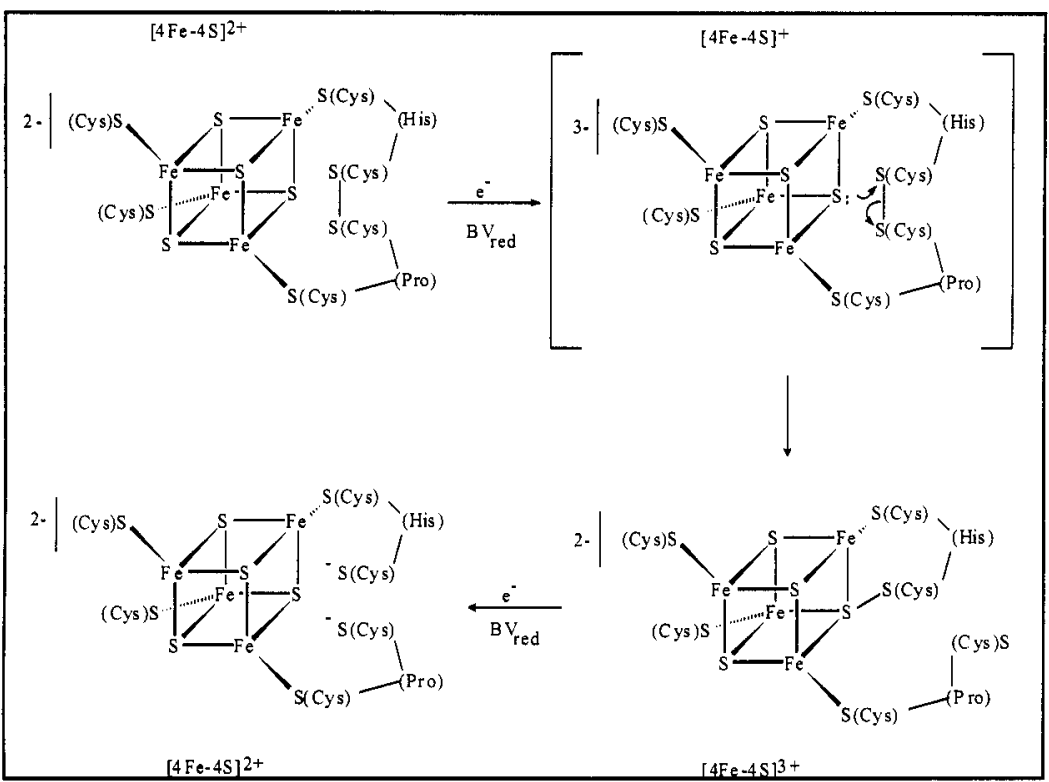

Fig. 5 Proposed scheme for the benzyl viologen-mediated 2-electron reduction of FTR disulfide coupled with oneelectron oxidation of the cluster to yield a species that is formally analogous to NEM-FTR, i.e. with one of the active site cysteines protected. With this in mind, Fig. 5 shows a viable scheme for the benzyl viologen-mediated twoelectron reduction of the active site disulfide in FTR. The scheme invokes a role for the cluster both in mediating electron transfer to the active site disulfide and in stabilizing the oneelectron reduced intermediate via covalent attachment of the electron transfer cysteine of the active site disulfide. Oneelectron reduction of the cluster leads to a transient formation of a $[4 \mathrm{Fe}-4 \mathrm{~S}]^{+}$ cluster which immediately cleaves the active site disulfide via nucleophilic attack involving an electron-rich $\mu_{3}-S^{2-}$. The resulting cluster is formally 
at the $[4 \mathrm{Fe}-4 \mathrm{~S}]^{3+}$ oxidation level, since 2-electrons have been withdrawn from the cluster in forming the covalent bond with Cys 84 of the active site disulfide. In terms of the Fe-S cluster, this one-electron reduced intermediate is analogous to NEM-FTR, which has Cys54 alkylated, and the heterodisulfide intermediate in the catalytic cycle, which has a disulfide linkage between Cys54 and one of the cysteines of the thioredoxin disulfide, see below. An alternative canonical form of the proposed one-electron reduced form is a $[4 \mathrm{Fe}-4 \mathrm{~S}]^{2+}$ cluster with a nearby cysteinyl radical. However, the spectroscopic data clearly argue for the unpaired electron density residing on the cluster. Nevertheless, this intermediate can at least formally be viewed as a cluster-stabilized thiyl radical. The second reducing equivalent from reduced benzyl viologen, then cleaves the novel $\mu_{3}-\mathrm{S}-\mathrm{S}$ (Cys) disulfide, at a potential typical for disulfide reduction, to yield the $[4 \mathrm{Fe}-4 \mathrm{~S}]^{2+}$ cluster and the fully reduced disulfide.

The above scheme provides a rationalization of the novel redox and spectroscopic properties of both FTR and NEM-FTR, and suggests a mechanism for biological disulfide reduction by an Fe-S cluster in sequential oneelectron steps that incorporates the thiol-disulfide interchange reaction that has been established for NAD(P)H-dependent flavin-containing disulfide oxidoreductases (4), see Fig. 6. We had previously proposed a similar scheme prior to establishing that NEM-FTR is indeed a stable analog of an enzymatic intermediate (8). After formation of the one-electron reduced form of FTR in which the electron-transfer cysteine (Cys84) is covalently attached to the cluster, the interchange cysteine (Cys54) attacks and cleaves the thioredoxin disulfide resulting in a heterodisulfide intermediate with oxidized-HiPIP-type cluster properties indistinguishable from those of NEM-FTR or the one-electron-reduced form of FTR. The second electron then cleaves the $\mu_{3}-\mathrm{S}-\mathrm{S}(\mathrm{Cys})$ disulfide restoring the cluster to the $[4 \mathrm{Fe}-4 \mathrm{~S}]^{2+}$ core oxidation state and freeing the electron-transfer cysteine to attack and cleave the heterodisulfide with reformation of the active site disulfide.

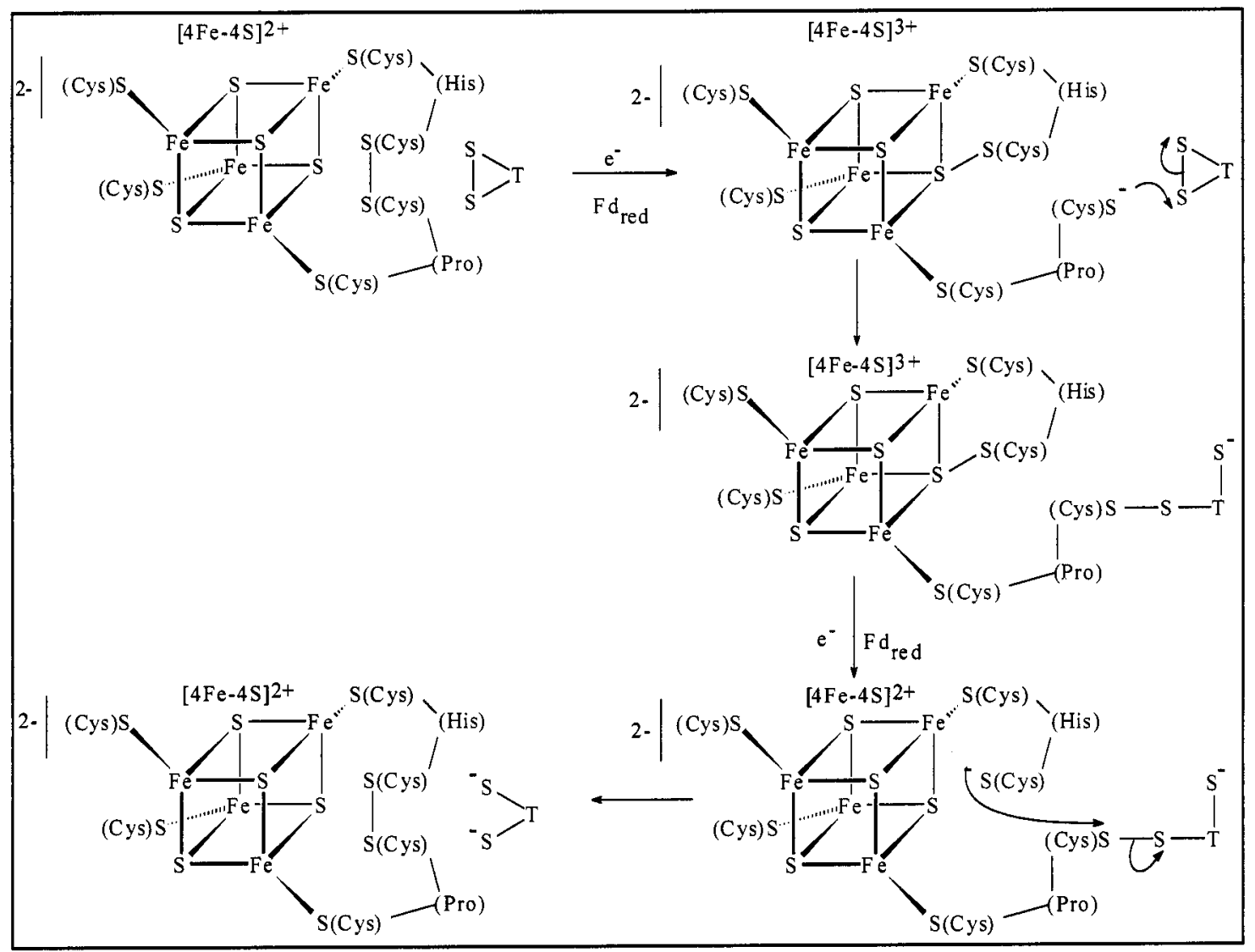

Fig. 6 Proposed mechanism for FTR

It should be stressed that there is as yet no direct evidence for the novel $\mu_{3}-\mathrm{S}-\mathrm{S}$ (Cys) disulfide linkage in NEM-FTR and the equivalent species in intermediate or one-electron reduced forms of FTR. In view of the anomalous properties of the Fe-S cluster in these species and the accessibility of the active site cysteines to alkylating agents, covalent attachment of the electron-transfer cysteine to the Fe-S cluster is likely to be an 
intrinsic part of the catalytic mechanism. However, we cannot rule out the possibility that this occurs at Fe to give a 5-coordinate Fe site. Attachment to a coordinating cysteine residue to form a cysteinyl disulfide and a non-cysteinyl-ligated $\mathrm{Fe}$ site is not a viable alternative, since this would be expected to yield a cluster at the $[4 \mathrm{Fe}-4 \mathrm{~S}]^{+}$rather than the $[4 \mathrm{Fe}-4 \mathrm{~S}]^{3+}$ oxidation level for the one-electron reduced species. Our reasons for favoring covalent attachment to the $\mu_{3}-\mathrm{S}^{2-}$ are fourfold. First, assuming that electron transfer to the active site disulfide occurs via the cluster, the bridging sulfides are the only nucleophilic sites on the cluster that can attack and cleave the active site disulfide. Second, RR studies with 457-nm excitation indicate a weak band at $538 \mathrm{~cm}^{-1}$ in NEM-FTR that is not present in FTR (9). This band is an excellent candidate for the S-S stretch of the $\mu_{3}-\mathrm{S}-\mathrm{S}(\mathrm{Cys})$ linkage with the enhancement coming via coupling with $\mathrm{Fe}-\mathrm{S}$ stretching modes. However, as yet it has not been possible to globally enrich samples with ${ }^{34} \mathrm{~S}$ in order to test this assignment. Third, the potential for one-electron reduction of NEM-FTR is in the range expected for disulfide reduction. Fourth, a similar slow-relaxing EPR signal $(g=2.09,2.00,1.98)$ has been observed in ferricyanide oxidized Azotobacter vinelandii $\mathrm{FdI}$ which has the $\mathrm{S}_{\gamma}$ of a free cysteine located $3.4 \AA$ from a $\mu_{3}-\mathrm{S}^{2-}$ of the $[4 \mathrm{Fe}-4 \mathrm{~S}]$ cluster (12)

\section{BIOTIN SYNTHASE}

Biotin synthase catalyzes the final step in the biosynthesis of biotin, i.e. the insertion of sulfur into dethiobiotin, see Fig. 7. Although the nature of the immediate sulfur donor remains elusive (13), the available evidence indicates that the formation of the thiol intermediate, or some derivative thereof, and possibly the

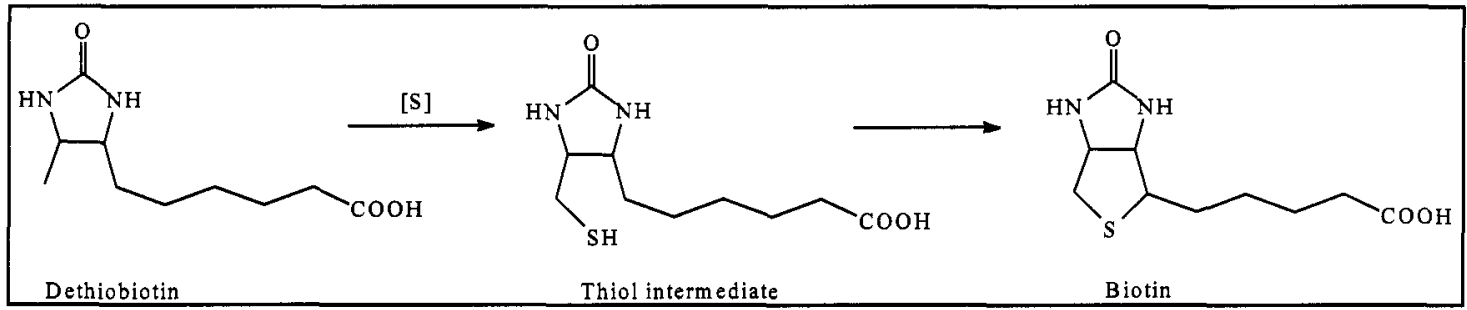

Fig. 7 Reaction catalyzed by biotin synthase

subsequent cyclization to form the thiophene ring, proceed by a radical mechanism with homolytic cleavage of the $\mathrm{C}-\mathrm{H}$ bond as the initial step $(14,15)$. Escherichia coli biotin synthase, or more specifically the bio $B$ gene product, was recently purified aerobically from recombinant strains and found to be a homodimer of $39-\mathrm{kDa}$ subunits with one $[2 \mathrm{Fe}-2 \mathrm{~S}]^{+}$cluster per monomer (16). In vitro activity requires $\mathrm{SAM}, \mathrm{NADPH}, \mathrm{Fe}^{2+}$ or $\mathrm{Fe}^{3+}$, an electron transport system which appears to be flavodoxin and flavodoxin reductase in vivo (17), and other proteins and/or cofactors in cell free extracts of bioB ${ }^{-}$strains of $E$. coli $(13,18)$. A well-defined in vitro assay with fully optimized activity has yet to be developed.

The obligate requirement for SAM and the presence of an Fe-S cluster has led to speculation that biotin synthase is a member of an emerging class of Fe-S enzymes with radical based mechanisms that include anaerobic ribonucleotide reductase (ARR) $(19,20)$, pyruvate formate-lyase (PFL) (21-23), and lysine 2,3aminomutase $(\operatorname{LAM})(24,25)$. In the case of ARR and PFL, the Fe-S cluster is associated with a homodimeric activating enzyme (ARR-AE and PFL-AE). However, the available spectroscopic and analytical evidence for these activases and for LAM indicate the presence of subunit bridging [4Fe-4S] clusters $(20,23,24)$, as opposed to the $[2 \mathrm{Fe}-2 \mathrm{~S}]$ clusters that are present in aerobically prepared samples of biotin synthase. Resolution of this puzzling observation has come from our recent spectroscopic studies of biotin synthase which have provided evidence for $2[2 \mathrm{Fe}-2 \mathrm{~S}]^{2+}+2 \mathrm{e}^{-}=[4 \mathrm{Fe}-4 \mathrm{~S}]^{2+}$ cluster interconversions (26).

Dithionite reduction of biotin synthase results in a gradual bleaching of the characteristic [2Fe-2S $]^{2}+$-cluster UV-visible absorption spectrum over a period of several hours. While analytical data indicate substantial cluster degradation, EPR and VTMCD studies showed the presence of $S=1 / 2[4 \mathrm{Fe}-4 \mathrm{~S}]^{+}$clusters $(0.1-0.3$ clusters per monomer based on EPR spin quantitations) as opposed to $S=1 / 2$ [2Fe-2S] $]^{+}$clusters. Moreover, if the reduction was carried out anaerobically in the presence $55 \%(\mathrm{v} / \mathrm{v})$ ethylene glycol, the absorption changes over a period of $2-3$ hours indicate almost quantitative reductive conversion of two $[2 \mathrm{Fe}-2 \mathrm{~S}]^{2+}$ clusters to yield one $[4 \mathrm{Fe}-4 \mathrm{~S}]^{2+}$ cluster. This cluster conversion is best illustrated by RR spectroscopy, see Fig. 8, since diamagnetic $[2 \mathrm{Fe}-2 \mathrm{~S}]^{2+}$ and $[4 \mathrm{Fe}-4 \mathrm{~S}]^{2+}$ clusters exhibit dramatically different $\mathrm{RR}$ spectra in the 


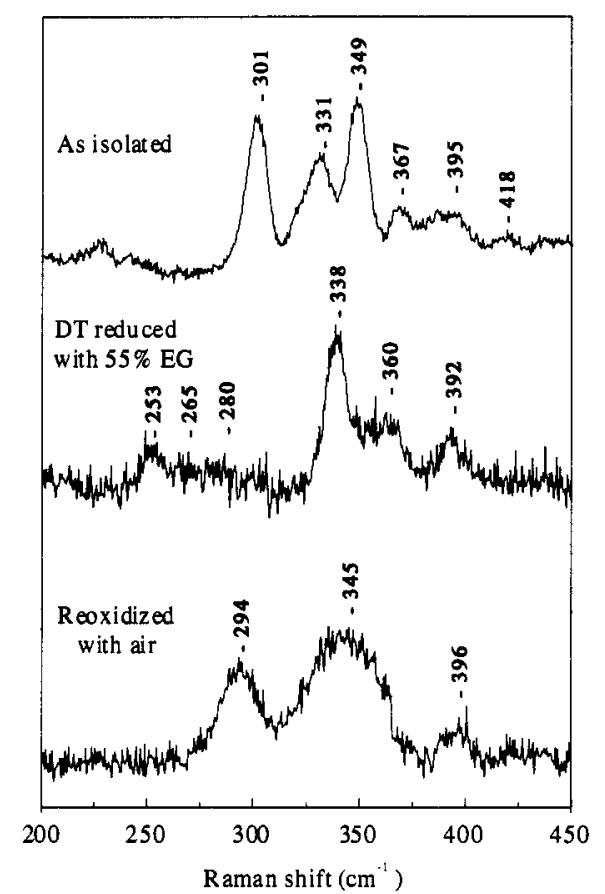

Fig. 8 RR spectra (459-nm excitation) of biotin synthase recorded at $25 \mathrm{~K}$.
Fe-S stretching region. Aerobically isolated biotin synthase exhibits a RR spectrum characteristic of biological [2Fe-2S $]^{2+}$ cluster. However, the frequency of the bands assigned to the mainly $\mathrm{Fe}-\mathrm{S}(\mathrm{Cys})$ stretching modes are indicative of a cluster with one oxygenic terminal ligand, e.g. similar to mutant $2 \mathrm{Fe}$ ferredoxins with serine replacing one of the coordinating cysteine ligands. After dithionite reduction in the presence of ethylene glycol, the bands associated with the $[2 \mathrm{Fe}-2 \mathrm{~S}]^{2+}$ cluster are completely lost and are replaced with the characteristic spectrum of a $[4 \mathrm{Fe}-4 \mathrm{~S}]^{2+}$ cluster with complete cysteinyl ligation. Since both absorption and analytical data indicate no significant loss of iron and no iron or sulfide were added during the reductive cluster conversion, we conclude that two [2Fe-2S] clusters have fused to give a single $[4 \mathrm{Fe}-4 \mathrm{~S}]$ cluster at the subunit interface. Furthermore the recent observation that this cluster conversion does not occur to a significant extent above

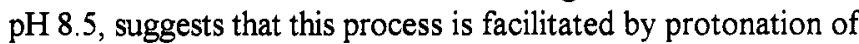
the oxygenic ligand. A tentative mechanism that summarizes the way we envisage this cluster conversion to occur is presented in Fig. 9. Precedent for the protonation of the oxygenic ligands to the reducible site of $[2 \mathrm{Fe}-2 \mathrm{~S}]^{+}$clusters comes from recent studies of the C56S and C60S mutant forms of Clostridium pasteurianum $2 \mathrm{Fe}$ ferredoxin which have cluster $\mathrm{pK}_{\mathrm{a}}$ values $\approx$ 9 in the reduced state (27).

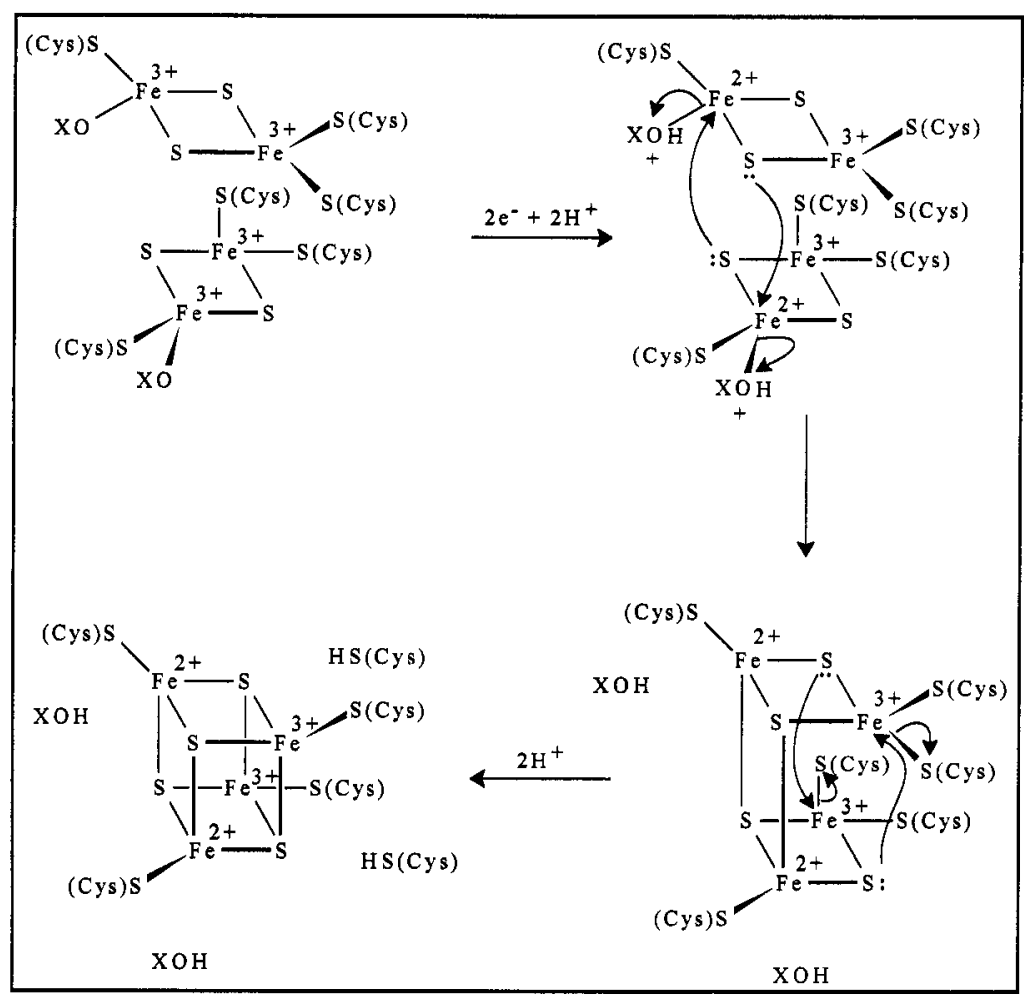

Fig. 9 Proposed scheme for the reductive dimerization of two [2Fe-2S] clusters to form a $[4 \mathrm{Fe}-4 \mathrm{~S}]$ cluster at the subunit interface in biotin synthase.
This cluster conversion is at least partially reversible, since a RR spectrum indicative of $[2 \mathrm{Fe}-2 \mathrm{~S}]^{2+}$ cluster is restored on aerial oxidation. The observation that the resulting $\mathrm{RR}$ spectrum is not the same as that of the as isolated enzyme does, however, suggest a difference in ligation for the $[2 \mathrm{Fe}-2 \mathrm{~S}]^{2+}$ clusters in asisolated and air-reoxidized samples. The interconvertibility of these two [2Fe-2S] forms is currently under investigation. Due to the lack of a fully optimized activity assay with well defined components, it has not been possible to correlate enzymatic activity with cluster type. However, since the [4Fe-4S]-containing enzyme is formed under anaerobic reducing conditions, it seems likely that this form of the enzyme is responsible for catalytic activity and that the [2Fe-2S]-containing forms correspond to $\mathrm{O}_{2}$-inactivated derivatives. A similar subunit-bridging [4Fe-4S] cluster, which also appears to undergo $[2 \mathrm{Fe}-2 \mathrm{~S}]-[4 \mathrm{Fe}-4 \mathrm{~S}]$ interconversions, is also found in PFL-AE (23), and the possible significance of this cluster interconversion and the role of the $[4 \mathrm{Fe}-4 \mathrm{~S}]$ cluster are discussed further below after reviewing the spectroscopic data for PFL-AE. 


\section{PYRUVATE FORMATE-LYASE ACTIVATING ENZYME}

PFL catalyzes the CoA-dependent cleavage of pyruvate to acetyl-CoA and formate. The elegant work of Knappe and coworkers has shown that the reaction requires the formation of a stable glycyl radical which participates in the homolytic C-C bond cleavage $(21,22,28)$. The mechanism of generating this catalytically essential radical involves an iron-dependent activating enzyme which requires reduced flavodoxin and SAM as co-substrates and pyruvate as a positive allosteric effector $(28,29)$. PFL-AE catalyzes the reductive cleavage of SAM to form methionine and 5'-deoxyadenosine (30), and although a 5'-deoxyadenosyl radical has yet to be observed as a transient intermediate in this or any other SAM-dependent enzyme, it is an attractive candidate for the $\mathrm{H}$-atom abstraction reaction to generate the active site glycyl radical (31).

Our recent spectroscopic studies have provided the first evidence that PFL-AE contains an Fe-S cluster (23). The recombinant enzyme, purified semi-anaerobically under an Ar atmosphere, has a red-brown color and is diamagnetic as evidenced by EPR and VTMCD studies in both as prepared and dithionite-reduced forms. As with biotin synthase, the identity and properties of the Fe-S clusters in these samples was revealed by the combination of absorption, $\mathrm{RR}$ and $\mathrm{Fe} / \mathrm{S}^{2-}$ analytical studies. After dithionite reduction, the absorption and $\mathrm{RR}$ spectra are characteristic of a $[4 \mathrm{Fe}-4 \mathrm{~S}]^{2+}$ cluster with complete cysteinyl ligation and the analytical data are consistent with $\sim 0.4$ [4Fe-4S] clusters per $28-\mathrm{kDa}$ monomer. However, the as-prepared sample was found to contain a mixture of $[2 \mathrm{Fe}-2 \mathrm{~S}]^{2+}$ and $[4 \mathrm{Fe}-4 \mathrm{~S}]^{2+}$ clusters. Indeed, the difference spectrum between the asprepared and dithionite-reduced forms, which reveals the RR spectrum of the $[2 \mathrm{Fe}-2 \mathrm{~S}]^{2+}$ cluster in PFL-AE, is remarkably similar to that of the $[2 \mathrm{Fe}-2 \mathrm{~S}]^{2+}$ clusters in air-reoxidized samples of biotin synthase. Although the $[4 \mathrm{Fe}-4 \mathrm{~S}]^{2+,+}$ cluster is not reducible with dithionite alone, it is reduced in the presence of SAM as evidenced by partial bleaching of the visible absorption and the appearance of a fast relaxing $S=1 / 2$ EPR signal $(g=2.01,1.89,1.88$, observable below $40 \mathrm{~K}$ ) accounting for $\sim 0.4$ spins per 28 -kDa monomer. The only way to reconcile these spectroscopic and analytical results is to invoke the same type of [2Fe-2S] $-[4 \mathrm{Fe}-$ 4S] cluster interconversion that occurs in biotin synthase. Hence the results indicate that the functional form of PFL-AE is a homodimer with one $[4 \mathrm{Fe}-4 \mathrm{~S}]^{2+,+}$ cluster at the subunit interface, that can undergo reversible oxidative conversion to $[2 \mathrm{Fe}-2 \mathrm{~S}]^{2+}$ clusters under conditions of incomplete anaerobicity. Moreover, the dependence on SAM for reduction, suggests that the $[4 \mathrm{Fe}-4 \mathrm{~S}]^{2+,}$ cluster is involved with the reductive cleavage of SAM to yield methionine and a 5'-deoxyadenosyl radical.

While there is still much work to be done to clarify the role of the subunit-bridging [4Fe-4S] clusters in SAMdependent Fe-S enzymes (biotin synthase, PFL-AE, ARR-AE, and LAM) and the physiological relevance (if any) of the $[2 \mathrm{Fe}-2 \mathrm{~S}]-[4 \mathrm{Fe}-4 \mathrm{~S}]$ cluster interconversion that has thus far only been established in biotin synthase and PFL-AE, our working hypotheses are summarized below. Only three cysteines are required to ligate the $[2 \mathrm{Fe}-2 \mathrm{~S}]$ cluster in each subunit and the $\mathrm{C}-\mathrm{X}_{3}-\mathrm{C}-\mathrm{X}_{2}-\mathrm{C}$ arrangement of cysteines that is found near the N-terminus in biotin synthase, PFL-AE and ARR-AE are clearly excellent candidates. (The sequence of

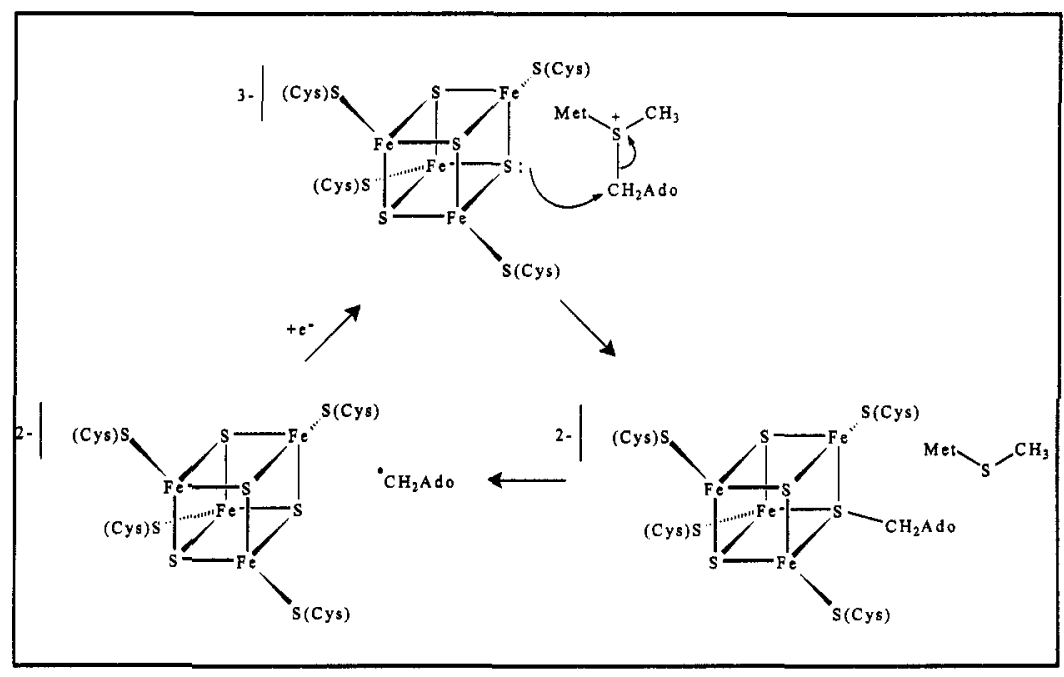

Fig. 10 Proposed mechanism for reductive cleavage of SAM by a $[4 \mathrm{Fe}-4 \mathrm{~S}]^{2+,+}$ cluster to yield methionine and a 5'-deoxyadenosyl radical
LAM has yet to be published). Only two of these three cysteines are required to ligate the [4Fe-4S] cluster at the subunit interface. Since the spectroscopic data for biotin synthase and PFL-AE indicate cysteinyl ligation at each Fe site of the subunitbridging [4Fe-4S] clusters and nucleophilic attack by the reduced $[4 \mathrm{Fe}-4 \mathrm{~S}]^{+}$ cluster is the most obvious method for cleaving the S-C bond in SAM, S-based cluster chemistry similar to that proposed above for FTR is currently our best working hypothesis for the role of the 
cluster in these SAM-dependent Fe-S enzymes, see Fig. 10. This mechanistic scheme is analogous to that proposed by Frey and Reed for LAM (25), although in this enzyme there is as yet no published evidence for a $[4 \mathrm{Fe}-4 \mathrm{~S}]^{+}$cluster in reduced samples. The mechanism involves nucleophilic attack by a $\mu_{3}-\mathrm{S}^{2-}$ of the $[4 \mathrm{Fe}-4 \mathrm{~S}]^{+}$cluster on SAM to form methionine and an intermediate adenosyl-[4Fe-4S] species that should have properties similar to that of a $[4 \mathrm{Fe}-4 \mathrm{~S}]^{3+}$ cluster, based on our studies of FTR. The $\mu_{3}-\mathrm{S}-\mathrm{C}$ bond would then be subject to homolytic cleavage since the cluster is a excellent one-electron acceptor, to yield a $[4 \mathrm{Fe}-4 \mathrm{~S}]^{2+}$ cluster and the 5'-deoxyadenosyl radical. The presence of $\mathrm{O}_{2}$ is likely to be particularly deleterious for this type of radical generation. Hence the oxygen-induced oxidative conversion to [2Fe-2S] clusters may be physiologically relevant in providing a means of shutting off enzyme activity without irreversible cluster degradation under periods of oxidative stress. In this connection, it is interesting to note that recent studies of the transcription factor FNR (fumarate nitrate reduction) have raised the possibility that the $\mathrm{O}_{2}$-sensing mechanism involves $\mathrm{O}_{2}$-induced degradation of a $[4 \mathrm{Fe}-4 \mathrm{~S}]^{2+}$ cluster to yield a $[2 \mathrm{Fe}-2 \mathrm{~S}]^{2+}$ cluster (32).

\section{ACKNOWLEDGMENTS}

We are indebted to our collaborators, Peter Schürmann and coworkers, University of Neuchâtel, Switzerland (spinach FTR), Dennis Flint and coworkers, E. I. du Pont de Nemours, Wilmington, USA (biotin synthase), and Joan Broderick and coworkers, Amherst College, Amherst, USA (PFL-AE), for supplying enzyme samples for spectroscopic investigations and for numerous insightful discussions. The spectroscopic studies summarized in this article were supported by a grant from NIH (GM-51962 to M.K.J.)

\section{REFERENCES}

1. M. K. Johnson. In Encyclopedia of Inorganic Chemistry Vol. 4 (R. B. King, ed.), pp. 1896-1915. Wiley, UK (1994).

2. H. Beinert, R. H. Holm and E. Münck. Science 277, 653-659 (1997).

3. D. B. Knaff and M. Hirasawa. Biochim. Biophys. Acta 1056, 93-125 (1991).

4. C. H. Williams Jr. In Chemistry and Biochemistry of Flavoenzymes Vol. 3 (F. Müller, ed.), pp. 121-211. CRC Press, Boca Raton (1992).

5. H. Iwadate, K. Yano, M. Kamo, L. Gardet-Salvi, P. Schürmann and A. Tsugita. Eur. J. Biochem. 223, $465-471$ (1994).

6. E. Falkenstein, A. Vonschaewen and R. Scheibe. Biochim. Biophys. Acta 1185, 252-254 (1994).

7. L.-P. Chow, H. Iwadate, K. Yano, M. Kamo, A. Tsugita, L. Gardet-Salvi, A.-L. Stritt-Etter and P. Schürmann. Eur. J. Biochem. 231, 149-156 (1995).

8. C. R. Staples, E. Ameyibor, W. Fu, L. Gardet-Salvi, A.-L. Stritt-Etter, P. Schürmann, D. B. Knaff and M. K. Johnson. Biochemistry 35, 11425-11434 (1996).

9. C. R. Staples, L. Gardet-Salvi, A.-L. Stritt-Etter, P. Schürmann, J. Telser, B. M. Hoffman, D. B. Knaff and M. K. Johnson. submitted for publication.

10. P. Schurmann, A.-L. Stritt-Etter and J. Li. Photosynth. Res. 46, 309-312 (1995).

11. Z. Salamon, G. Tollin, M. Hirasawa, L. Gardet-Salvi, A.-L. Stritt-Etter, D. B. Knaff and P. Schurmann. Biochim. Biophys. Acta 1230, 114-118 (1995).

12. Z. Hu, D. Jollie, B. K. Burgess, P. J. Stephens and E. Münck. Biochemistry 33, 14475-14485 (1994).

13. I. Sanyal, K. J. Gibson and D. H. Flint. Arch. Biochem. Biophys. 326, 48-56 (1996).

14. F. B. Marti. Diss. E.T.H. Zurich, Nr 7236 (1983).

15. E. Jestin, F. Moreau, D. Florentin and A. Marquet. Bioorg. Med. Chem. 4, 1065-1075 (1996).

16. I. Sanyal, G. Cohen and D. H. Flint. Biochemistry 33, 3625-3631 (1994).

17. O. Ifuku, N. Koga, S.-I. Haze, J. Kishimoto and Y. Wachi. Eur. J. Biochem. 224, 173-178 (1994).

18. O. M. Birch, M. Fuhrmann and N. M. Shaw. J. Biol. Chem. 270, 19158-19165 (1995).

19. P. Reichard. (1993) J. Biol. Chem. 268, 8383-8386 (1993).

20. S. Ollangnier, E. Mulliez, J. Gaillard, R. Eliasson, M. Fontecave and P. Reichard. (1996) J. Biol. Chem. 271, $9410-9416$ (1996).

21. J. Knappe, F. A. Neugebauer, H. P. Blaschkowski and M. Gänzler. Proc. Natl. Acad. Sci. USA 81, 1332-1335 (1984).

22. J. Knappe and G. Sawers. FEMS Microbiol. Rev. 75, 383-398 (1990).

23. J. B. Broderick, R. E. Duderstadt, D. C. Fernandez, K. Wojtuszewski, T. F. Henshaw and M. K. Johnson. J. Am. Chem. Soc.119, 77396-77397 (1997).

24. R. M. Petrovich, F. J. Ruzicka, G. H. Reed and P. A. Frey. Biochemistry 31, 10774-10781 (1992).

25. P. A. Frey and G. H. Reed. Adv. Enzymol. Relat. Areas Mol. Biol. 66, 1-39 (1993).

26. E. C. Duin, M. E. Lafferty, B. R. Crouse, R. M. Allen, I. Sanyal, D. H. Flint and M. K. Johnson. Biochemistry 36, in press.

27. M. K. Johnson, E. C. Duin, B. R. Crouse, M.-P. Golinelli and J. Meyer. In Spectroscopic Methods in Bioinorganic Chemistry (E. I. Solomon and K. O. Hodgson, eds.), ACS Books Symposium Series, in press.

28. A. F. V. Wagner, M. Frey, F. A. Neugebauer, W. Schäfer and J. Knappe Proc. Natl. Acad. Sci. USA 89, 996-1000 (1992).

29. K. K. Wong, B. W. Murray, S. A. Lewisch, M. K. Baxter, T. W. Ridky, L. Ulissi-DeMario and J. W. Kozarich. Biochemistry 32, 14102-14110 (1993).

30. J. Knappe and T. Schmidt. Biochem. Biophys. Res. Commun. 71, 1110-1117 (1976).

31. M. Frey, M. Rothe, A. F. Volker Wagner and J. Knappe. J. Biol. Chem. 269, 12432-12437 (1994).

32. N. Khoroshilova, C. Popesu, E. Münck, H. Beinert and P. J. Kiley. Proc. Natl. Acad. Sci. USA 94, 6087-6092 (1997). 